\title{
Ultrafast Vibrational Spectroscopy of the Flavin Chromophore
}

Kondo et al (jp0650735)

Supplementary data

In the main text reference is made to DFT calculations using the Gaussian 03 software. In particular we make use of the dipole orientation in interpreting the polarization resolved data. Due to space limitations more detailed descriptions of the output are presented here. None of the frequencies presented below have been scaled

In Figure s1 the calculated IR spectrum is shown.

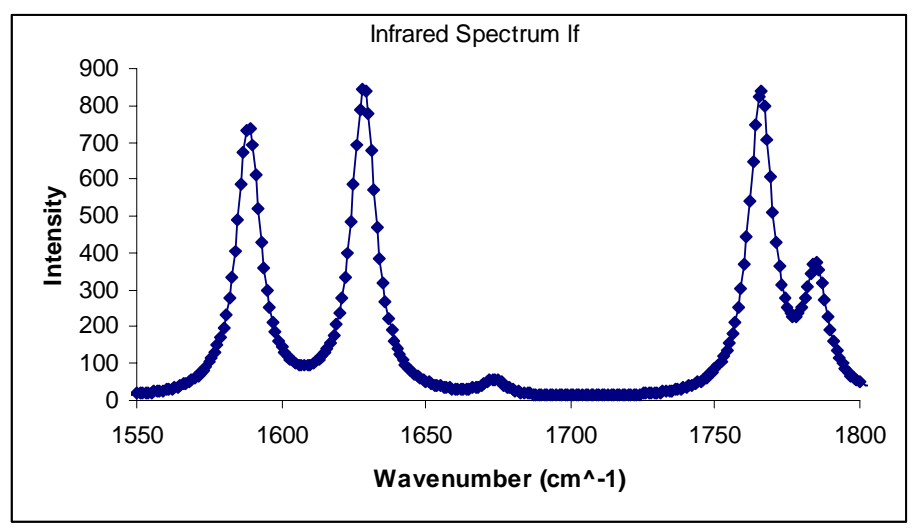

Fig s1. The DFT calculated IR spectrum of Lf showing the five most relevant modes. For comparison with experiment a scaling factor of 0.96 is usually applied

The two highest frequency modes correspond to the two carbonyl stretch modes at $1795 \mathrm{~cm}^{-1}$ (Fig s2)

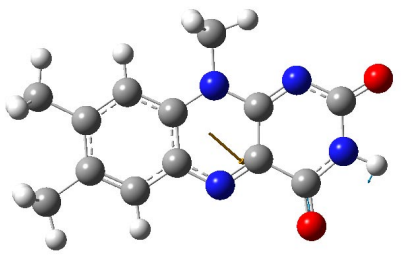

Fig s2. The highest frequency $C=O$ stretch of $L f$. The transition dipole derivative and bond vectors are shown

and $1766 \mathrm{~cm}^{-1}$ (Fig s3). As can be seen the calculated transition dipole derivative does not lay exactly along the bond in either case, indicating the involvement of other bonds in the normal mode, most notably the $\mathrm{NH}$ wag of the adjacent $\mathrm{NH}$ group.

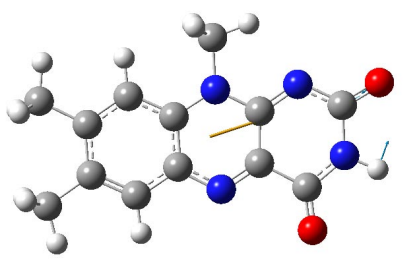

Fig s3. The second highest frequency $C=O$ stretch of $L f$. The transition dipole derivative and bond vectors are shown 
The weak mode at $1673 \mathrm{~cm}^{-1}$ has an out of plane dipole derivative and is localized on the phenyl ring and the central CN stretch.

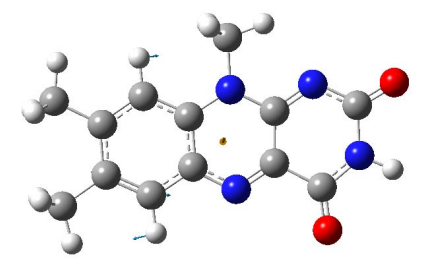

Fig s4. The $1673 \mathrm{~cm}^{-1}$ mode of Lf. The transition dipole derivative is out of plane.

The intense $1628 \mathrm{~cm}^{-1}$ mode involves a significant $\mathrm{CN}$ stretch component

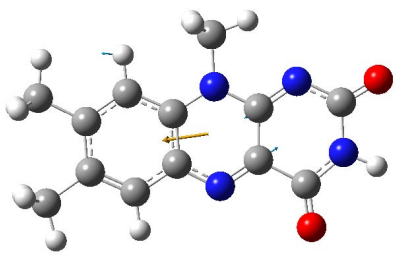

Fig s5. The $1628 \mathrm{~cm}^{-1}$ mode of Lf. The transition dipole derivative and bond displacements are shown.

Finally the lowest frequency $\left(1589 \mathrm{~cm}^{-1}\right)$ mode in our range of interest is delocalized over the phenyl ring and $\mathrm{CN}$ stretch

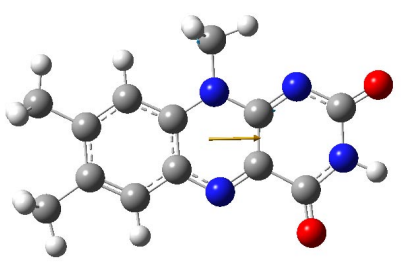

Fig s5. The $1589 \mathrm{~cm}^{-1}$ mode of Lf. The transition dipole derivative and bond displacements are shown. 